\title{
Detección de Posición Angular de Embarcaciones, utilizando Técnicas de Visión Computacional y Redes Neurales Artificiales
}

\author{
Vilson B. Mendes ${ }^{(1)}$, Fabiana R. Leta ${ }^{(2)}$, Aura Conci $^{(2)}$ y Laercio B. Gonçalves ${ }^{(3)}$ \\ (1) Inst. de Investigación de la Marina del Brasil, R. Ipiru 2, 21931-090, Rio de Janeiro, RJ-Brasil \\ (2) Univ. Federal Fluminense, Programa de Post-Grado en Ingeniería Mecánica y Inst. de \\ Computación, R. Passo da Patria, 156, 24210-240, Niteroi, RJ-Brasil \\ (3) Dpto. de Computación, CEFET-RJ, Av. Maracanã 229, 20271-110, Rio de Janeiro, RJ-Brasil
}

Recibido Ene. 07, 2010; Aceptado Feb. 22, 2010; Versión Final recibida Abr. 16, 2010

\begin{abstract}
Resumen
Este trabajo presenta un sistema de detección de posición angular de buques, utilizando técnicas de extracción de características en imágenes digitales y redes neurales artificiales. Se utilizan imágenes de embarcaciones militares generadas gráficamente. Se realizaron diferentes pruebas usando redes neuronales artificiales aplicadas al conjunto de características geométricas. Los resultados de las pruebas comprueban la importante contribución de la utilización de algoritmos de reconocimiento en la determinación de posicionamiento angular de embarcaciones, independiente del alejamiento del observador. Los resultados favorecen aplicaciones futuras en el seguimiento de buques (tracking) utilizando imágenes infrarrojas.
\end{abstract}

Palabras clave: tracking, redes neuronales, visión computacional, imágenes infrarrojas

\section{Target Angular Position Detection using Computer Vision Techniques and Artificial Neural Networks}

\begin{abstract}
This paper presents a system for detecting angular position of targets, using feature extraction techniques in digital imaging and artificial neural networks. Military ships images graphically generated by three-dimensional solid modeling software are used. Several tests using artificial neural networks applied to the set of geometric features were performed. The results show the important contribution of recognition algorithms in determining the ship angular position, regardless of their distance from the observer. The results encourage future applications for tracking targets using infrared images.
\end{abstract}

Keywords: seguimiento, artificial neural networks, computer vision, infrared images 


\section{INTRODUCCIÓN}

El desarrollo de metodologías para sistemas de reconocimiento de embarcaciones militares a través de imágenes infrarrojas viene despertando gran interés en aplicaciones tanto en el sector militar como en el comercial, pues a pesar de la eficiencia de estos sistemas estar sujeta a diversos factores (Behrooz, 2001), como variaciones climáticas, se trata de un sistema pasivo, o sea, en que la recepción no necesita de una inducción, y por tanto son más seguros en relación a una contramedida electrónica. Por eso, este tipo de imagen viene haciéndose cada vez más común en aplicaciones que incluyen seguridad, en especial en el campo militar.

Una imagen infrarroja es originada a partir del resultado de adquisición de la radiación térmica de una escena, que puede ser producida sin cualquier luz visible. En esta adquisición, es producido un mapa bidimensional representando parámetros como la variación de temperatura, de irradiación y de reflexión (Neves, 2003). Por eso, así como una imagen digital monocromática, la estructura de datos de una imagen infrarroja puede ser considerada como una matriz $m \times n$ donde los índices de línea y columna identifican un punto en la imagen y el valor numérico del elemento correspondiente en la matriz está asociado a la temperatura medida en este punto (Deer, 1997). Para extraer informaciones de este tipo de imagen se utilizan los mismos procesos de tratamiento de imágenes, como filtración, segmentación entre otros.

En tácticas de guerra, además del reconocimiento de embarcaciones, existe aún el interés en el acompañamiento del blanco y, en función de las bajas velocidades de las embarcaciones, estas presentan poco desplazamiento en relación al referencial de las imágenes capturadas. Además de eso, es común que el sistema pierda el acompañamiento del blanco cuando su silueta es interceptada por siluetas de embarcaciones semejantes con movimiento en direcciones diferentes. La detección de la dirección de la embarcación (Fig. 1) posibilita delinear previamente su comportamiento en cuanto al movimiento, evitándose la pérdida del acompañamiento del blanco, a partir de la obtención del ángulo y del sentido de desplazamiento de las embarcaciones. Así, el artículo presenta un estudio exploratorio, en que se utilizan aplicaciones técnicas de hecho bien conocidas de extracción de características y métodos de decisión como forma de validar una metodología que sea capaz de determinar variaciones angulares tridimensionales de objetos.

Una de las mayores dificultades en la detección del ángulo del sentido de desplazamiento de una embarcación es que las embarcaciones presentan siluetas semejantes para diferentes posiciones angulares y de ampliación. De esta forma, en este artículo se presenta una metodología, utilizando técnicas de extracción de características en imágenes digitales y redes neurales artificiales, para el reconocimiento de la dirección de embarcaciones, considerando diferentes grupos de características extraídas de las imágenes. Reconocimiento de navíos a través de curves principales, presentado por Fernandez (2005), o a través de momentos invariantes, presentado por Alves (2001) o de aeronaves a través de momentos invariantes wavelet, presentado por Feng (2008) tratan del problema considerando proyecciones sin alteraciones angulares. En ese sentido, es analizado el comportamiento de diferentes modelos de redes neurales teniendo como datos de entrada un conjunto de ocho características geométricas.

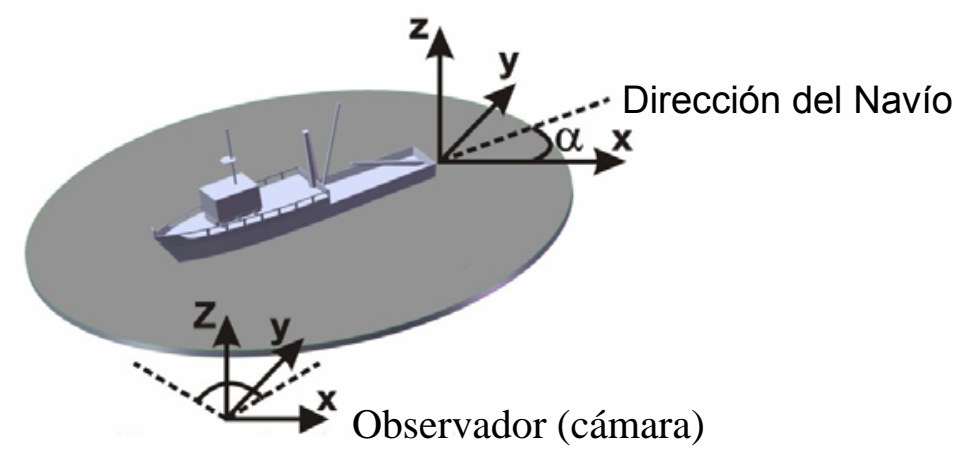

Fig. 1: Ángulo de la dirección de la embarcación en relación al referencial del observador. 
Para determinar la posición angular, consideramos que la dirección del navío se ajusta a su dimensión axial más larga y se coloca en su sistema inercial. La posición angular es el ángulo entre el plano xz (plan de adquisición de la imagen) y el plano wz (alineado a la nave de dirección), ambos con dirección z perpendiculares al mar. El plano de adquisición de la imagen se coloca en la referencial del la cámara y es importante no confundir con el referencial de la imagen. El referencial de la imagen está relacionado con la proyección de los objetos después de la adquisición. El referencial de la cámara se refiere la posición de la cámara en un segundo navío.

Debido a la dificultad de la obtención de un conjunto significativo de imágenes infrarrojas de embarcaciones con variaciones regulares de posición angular y ampliación, para extraer las características, fueron utilizados modelos tridimensionales de las embarcaciones construidos computacionalmente en el software de modelaje de sólidos SolidWorks $®$. Las características obtenidas fueron utilizadas en los entrenamientos de los modelos de redes neurales. Para este estudio exploratorio, el objetivo es conseguir informaciones importantes sobre el caso en estudio, como la verificación de la posibilidad de mapearse una función que caracteriza la salida y también estimar cuanto esta varia para diferentes plataformas.

\section{SISTEMA DE VISIÓN COMPUTACIONAL PARA DETECCIÓN DE BLANCOS}

Un sistema de Visión Computacional tiene como meta obtener, a partir de una imagen digital, informaciones geométricas, topológicas o físicas sobre el escenario u objetos que componen esta imagen, para realizar algún proceso decisorio. De acuerdo con su aplicación, estas informaciones pueden permitir el reconocimiento de patrones, la clasificación de objetos, el movimiento de robots, etc. También es pertinente comentar que análisis de técnicas de tratamiento de la imagen, preprocesamientos, deben ser consideradas para situaciones de imágenes reales en la contribución de uso de técnicas de Visión Computacional como alternativa para la detección de ángulos de objetos en imágenes infrarrojas. Considerando que existen varias etapas en un sistema de Visión Computacional genérico (Conci et al, 2008), conforme puede ser visto en la Fig. 2, el preprocesamiento está relacionado a las etapas de restauración, realce y segmentación de la imagen, y consiste en un proceso de suma importancia debido a la gran variedad de técnicas que explora esta redundancia para recuperar informaciones contenidas en la imagen o realizar una mejoría en la calidad de la imagen.

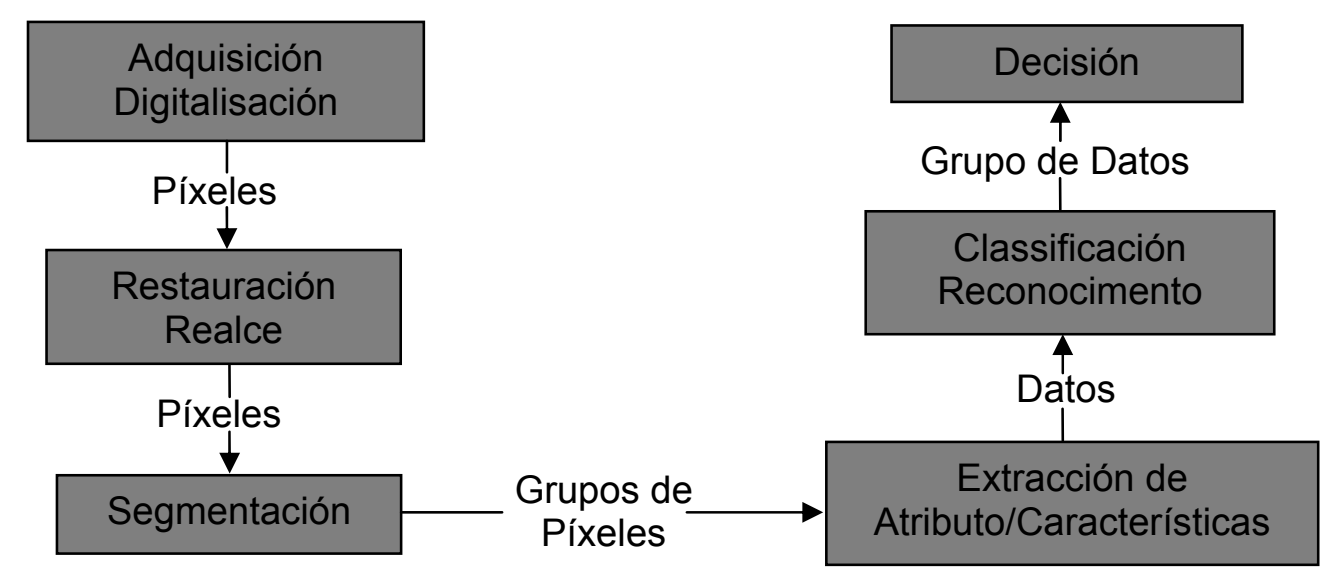

Fig. 2: Etapas de un sistema de VC genérico (Conci et al, 2008).

Tales técnicas ejecutan transformaciones en la imagen, procurando mejorar su la calidad, de manera que se pueda facilitar el proceso de extracción de parámetros intrínsecos, como discontinuidades de superficies, distancia, orientación de superficies, entre otros. Trabajos sobre técnicas de preprocesamiento en imágenes infrarrojas son presentados por Neves (2005) y Carvalho (2008), donde se destaca que la elección de las técnicas de restauración/realce y de segmentación es fundamental para un sistema de reconocimiento de objetos, pues, si son inadecuadas, pueden acrecentar o eliminar informaciones en la imagen, lo que puede ocasionar resultados incorrectos en la etapa de extracción de características (John Russ, 1998). En este artículo, fueron utilizados modelos de embarcaciones para análisis, hecho que elimina la aplicación de tales técnicas usuales en imágenes 
obtenidas por sistemas de captura. De esta forma se consideran las etapas de extracción de atributo/características y de clasificación/reconocimiento. Para el estudio exploratorio presentado, se desea validar la metodología en que uno de los objetivos fue evaluar la posibilidad de mapearse una función de caracterización da salida.

\section{Extracción de Características}

Dentro del universo del área de reconocimiento, en términos de aplicación, la Visión Computacional tiene como objetivo reconocer cualquier tipo de padrón detectable, y en los casos de identificación de las embarcaciones y/o determinación del ángulo del sentido de desplazamiento, esta área se puede restringir a la extracción de características de forma de objetos (Ruiz, 2001).

Específicamente en relación al caso en cuestión, la extracción se resume a la obtención de las características que sean invariables en el espacio geométrico. Sin embargo, se sabe que una embarcación es un objeto con volumen que varía en un espacio tridimensional y además que las imágenes obtenidas por cámaras digitales son representaciones bidimensionales de objetos, considerando una proyección, en general, perspectiva. De este modo, se propone que, cuando sea identificada la embarcación, utilizando un conjunto de características extraídas de estas representaciones bidimensionales, sea posible obtener información sobre el ángulo de dirección.

Por lo tanto, teniendo como base objetos destacados en imágenes monocromáticas binarias, se pueden citar diferentes tipos de características posibles de ser obtenidas, siendo que este trabajo está relacionado a algunas características dimensionales e inerciales, considerando el objetivo en pauta.

\section{Características Geométricas}

Las características geométricas en imágenes de objetos bidimensionales son invariantes en lo que se refiere a la rotación y traslación del objeto en relación a un referencial en la imagen. Esto significa que independiente de la posición del objeto en la imagen, se tiene los mismos valores de tales características, siendo, por lo tanto, importantes para la función de reconocimiento en un sistema de visión.

Considerando que después del pre-procesamiento de la imagen de un objeto (embarcación) se tiene la embarcación en negro y el fondo en blanco, el área de la embarcación puede ser obtenida fácilmente por la suma de píxeles negros (puntos en la imagen) pertenecientes al objeto (Ruiz, 2001). Al considerarse el objeto inscrito en un rectángulo tanto en el sentido del referencial de la imagen como en el sentido de alargamiento, pueden determinarse características como el área del rectángulo envolvente, el ángulo del alargamiento, la mayor y la menor dimensión del rectángulo alineado al referencial de la imagen, o del rectángulo alineado al sentido del largo $\left(L_{\max }\right.$ y $\left.H_{\max }\right)$, entre otras. Es importante resaltar que esas características dependen de la forma como la imagen del objeto fue capturada, pues las dimensiones del objeto varían en relación a la distancia entre el objeto y la cámara, además de sufrir influencia en relación al ángulo entre el referencial de la cámara y la dirección de la embarcación ( $\alpha$ ), conforme fue presentado en la Fig. 1.

Así como el área, el perímetro del objeto puede ser obtenido a través de la suma de los puntos del objeto que son vecinos al fondo. Otras características como excentricidad y ángulo de orientación son obtenidas en función de algunos momentos geométricos (Ruiz, 2001).

Las llamadas características inerciales se basan en las teorías de momento de inercia. Conforme Alves (2001) momentos han sido utilizados para reconocimiento de patrones en imágenes bidimensionales desde la década de 1960. Existen varios tipos de momentos, pero los invariables, en relación a la rotación, translación y escala, están relacionados con los momentos geométricos, que son basados en los conceptos de inercia de los cuerpos rígidos.

Para realizar la extracción de las características de objetos, se considera una imagen discreta con $N \times M$ píxeles en dos tonos de gris (negro y blanco), donde el color negro pertenece al objeto. Conci (2008) presenta los momentos geométricos de orden $(p+q)$ calculados a partir de la ecuación 1. 
$m_{p q}=\sum_{k=1}^{n} B\left(i_{k}, j_{k}\right)\left(i_{k}\right)^{p}\left(j_{k}\right)^{q} p, q=0,1,2, \ldots$

Siendo $n$ el número de píxeles negros y $B(i, j)$ un píxel negro de coordenadas $i$ y $j$. En esta anotación, el momento geométrico de orden cero representa el área del conjunto $B$. A partir de las relaciones entre los momentos de orden 0 y 1 se puede determinar el centroide en coordenadas $\left(i_{0}, j_{0}\right)$ del conjunto $B$, que es una característica importante del objeto en la imagen (ecuación 2 y 3 ).

$$
\begin{aligned}
& i_{0}=\sum_{k=1}^{n} B\left(i_{k}, j_{k}\right) i_{k} / \sum_{k=1}^{n} B\left(i_{k}, j_{k}\right)=m_{10} / m_{00} \\
& j_{0}=\sum_{k=1}^{n} B\left(i_{k}, j_{k}\right) j_{k} / \sum_{k=1}^{n} B\left(i_{k}, j_{k}\right)=m_{01} / m_{00}
\end{aligned}
$$

Los momentos centrales de inercia son calculados a partir de la translación de los momentos geométricos de objetos en relación al origen del sistema de coordenadas, para las coordenadas del centroide de los objetos, de forma que el objeto pueda ser descrito en relación a un sistema de coordenadas que pase por su centroide (ecuación 4). Puesto que las coordenadas del centroide $\left(i_{0}, j_{0}\right)$ ocupan siempre la misma posición relativa con respecto a todos los puntos del objeto, los momentos centrales no varían ante traslaciones y rotación de los objetos.

$$
\mu_{p q}=\sum_{k=1}^{n} B\left(i_{k}, j_{k}\right)\left(i_{k}-i_{0}\right)^{p}\left(j_{k}-j_{0}\right)^{q} B\left(i_{k}, j_{k}\right) p, q=0,1,2, \ldots
$$

A partir de la ecuación 4 se obtienen los momentos centrales para todas las órdenes $o(o=p+q)$. Los más utilizados son:

$$
\begin{array}{ll}
\mu_{0,0}=m_{0,0} & \text { (área) } \\
\mu_{0,1}=\mu_{1,0}=0 & \text { (momentos estáticos) } \\
\mu_{0,2}=m_{0,2}-i_{0} m_{0,1} & \text { (momentos de inercia) } \\
\mu_{2,0}=m_{2,0}-i_{0} m_{1,0} & \text { (momentos de inercia) } \\
\mu_{1,1}=m_{1,1}-j_{0} m_{1,0} & \text { (producto de inercia) }
\end{array}
$$

Considerando $\mu_{2,0}, \mu_{0,2}$ y $\mu_{1,1}$, se puede determinar el ángulo $\theta$ (Fig. 3) que identifica la orientación de los ejes principales a través de la ecuación 5 :

$\tan 2 \theta=2 \mu_{1,1} /\left(\mu_{2,0}-\mu_{0,2}\right)$

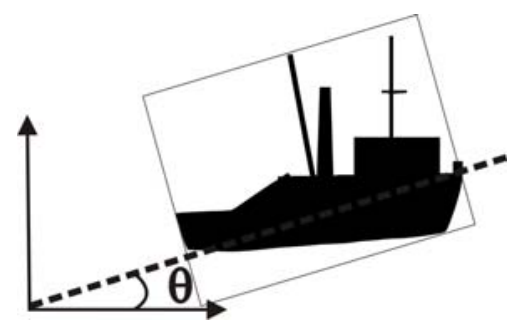

Fig. 3: Orientación.

Es importante observar que este ángulo es obtenido considerando el referencial bidimensional de la imagen, o sea, no es representativo de la posición angular espacial del objeto ( $\alpha$ ), que es el objetivo de este trabajo.

Otra característica utilizada fue la excentricidad (E) que es definida como la división entre el tamaño del menor y mayor eje, caracterizando como el objeto está distribuido espacialmente entre sus ejes. Esta medida también puede ser calculada en función de los momentos centrales conforme muestra la ecuación 6. 
$E=\frac{\left(\mu_{02}-\mu_{20}\right)^{2}+4 \mu_{11}}{\text { Area }}$

Otra forma de obtenerse la característica referente a la excentricidad puede ser a través de la elipse ajustada que es aquella que tiene los momentos de inercia iguales a los del objeto. Considerando la orientación $\theta$, los momentos de inercia máximo y mínimo de la elipse pueden ser calculados conforme las ecuaciones 7 y 8 .

$$
\begin{aligned}
& I_{\text {min }}^{\prime}(\theta)=\sum_{i}^{n} \sum_{j}^{n}\left[\left(i-i_{0}\right) \cos \theta-\left(j-j_{0}\right) \operatorname{sen} \theta\right]^{2} \\
& I_{\text {max }}^{\prime}(\theta)=\sum_{i}^{n} \sum_{j}^{n}\left[\left(i-i_{0}\right) \operatorname{sen} \theta+\left(j-j_{0}\right) \cos \theta\right]^{2}
\end{aligned}
$$

De esta forma, pueden obtenerse dos parámetros correspondientes a la excentricidad conforme presentan las ecuaciones 9 y 10 .

$$
\begin{aligned}
& E_{1}=\frac{I_{\text {max }}^{\prime}(\theta)}{I_{\text {min }}^{\prime}(\theta)} \\
& E_{2}=\frac{A}{B}
\end{aligned}
$$

Donde $A$ y $B$ son los largos de los semi-ejes mayor y menor de la elipse, y pueden ser obtenidos a través de las ecuaciones 11 y 12.

$$
\begin{aligned}
& A=\left(\frac{4}{\pi}\right)^{\frac{1}{4}}\left[\frac{I_{\text {max }}^{\prime}}{I_{\text {min }}^{\prime}}\right]^{\frac{1}{8}} \\
& B=\left(\frac{4}{\pi}\right)^{\frac{1}{4}}\left[\frac{I_{\text {min }}^{\prime}}{I_{\text {max }}^{\prime}}\right]^{\frac{1}{8}}
\end{aligned}
$$

\section{Reconocimiento}

De acuerdo con Alves (2001), técnicas de árboles de decisión teniendo como entradas características que sean invariables en el espacio pueden ser utilizadas en la identificación y reconocimiento de embarcaciones. Este trabajo visa comprobar la utilización de esas características en la obtención del ángulo del sentido de desplazamiento.

El proceso de rotular un determinado objeto genérico, con base en un conjunto de características previamente establecidas es denominado de Reconocimiento o Clasificación (Chang, 1998).

La mayoría de los problemas de clasificación de patrones de interés real posee dos fases distintas. La primera, en general llamada de in sample o de entrenamiento (aprendizaje), es ejecutada a partir del banco de datos existente. La segunda fase, conocida como out sample o generalización, es ejecutada para los datos que no fueron utilizados en el entrenamiento. La segunda fase permite la evaluación con los datos 'nuevos', del modelo producido (explícitamente o no) en la primera fase. Lo que se desea, fundamentalmente, es extraer las características generales del ambiente, para posteriormente utilizarlas en la toma de decisión en datos nuevos, aún no presentados al sistema.

Los métodos de clasificación consisten en técnicas capaces de distinguir objetos de diferentes clases. Esa diferenciación es eficiente cuando la imagen es compuesta por $n$ objetos pertenecientes a $n$ clases diferentes. Las técnicas de clasificación pueden ser abordadas de dos formas: 
supervisada y no supervisada. Siendo que, para reconocimiento del ángulo del sentido de desplazamiento de las imágenes de las embarcaciones, a clasificación será supervisada.

\section{Clasificación Supervisada}

La clasificación supervisada es aquella en que un conjunto de objetos conocidos pertenecientes a diferentes clases es analizado, escogiéndose los parámetros ideales para la separación de las clases. Estos parámetros definen la función discriminante que separa las diversas clases. La clasificación supervisada puede ser hecha por distribución estadística o distribución libre. La primera se basa en modelos de distribución de probabilidades. La segunda no requiere conocimiento previo de funciones o distribución de probabilidades, se basa en deducción y heurística. Como ejemplo de método de clasificación por distribución libre que permite un rápido progreso hasta una decisión final, puede citarse a las Redes Neurales Artificiales (Freeman, 1992), que fueron utilizadas en este trabajo en el reconocimiento del ángulo del sentido de desplazamiento.

Una red neural artificial puede ser considerada una técnica computacional en la cual se tiene la construcción de un modelo matemático de un sistema neural biológico simplificado, con capacidad de aprendizaje, generalización, asociación, clasificación, etc. Las redes neurales intentan "aprender" las características de patrones a través de un proceso de repetidas presentaciones de los datos a la red. De esa forma, una red neural busca relaciones, construye modelos de forma automática y los corrige de modo a minimizar su error (Haykin, 1996). Neurona artificial es un elemento de procesamiento de información esencial para la operación de las llamadas Redes Neurales. El modelo de una neurona artificial es presentado en la Fig. 4 y consiste de $m$ entradas ponderadas por los pesos $W_{k 1 . . m}$ y entonces sumadas en una unión aditiva. Esos pesos son conocidos como pesos sinápticos y son responsables por la capacidad de modelar y aprender de una red neural. Una función de activación se coloca en seguida al sumador, que limitará el intervalo permisivo de salida de la neurona a la que se refiere. El modelo prevé aún un ajuste denominado bias que tiene el efecto de aumentar o disminuir el valor aplicado a la función de activación (Gonçalves Filho, 1996).

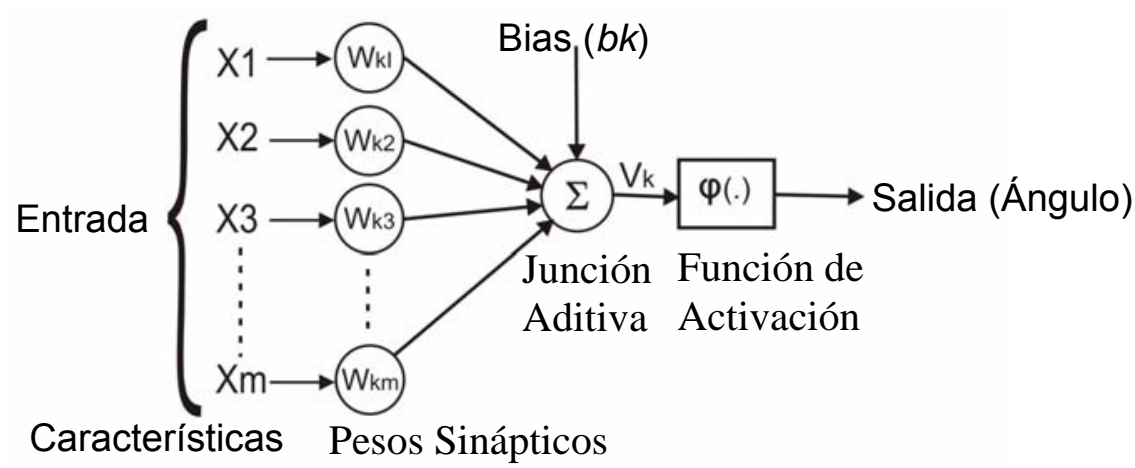

Fig. 4: Modelo de neurona artificial no lineal (Gonçalves Filho, 1996).

Después de definir la característica de una neurona, la arquitectura de una red neural puede ser definida uniendo neuronas entre si a través de los pesos sinápticos. La configuración presentada en la Fig. 5 es la de una red neural de múltiples capas conocida como "Perceptrons de múltiples capas" o MLP (multilayer perceptrons).

De modo general, en la tarea de clasificación de patrones, la arquitectura de la red neural recibe atributos como entrada de la primera capa de la red neural. Los atributos objetivos (clases) son modelados por la capa de salida de la red neural. De ese modo el algoritmo puede estimar cuanto la salida deseada está distante de la salida real (Valencia, 1999).

\section{METODOLOGIA}

Conforme mencionado anteriormente, modelos sólidos fueron construidos computacionalmente con la finalidad de generar las diferentes vistas para ser utilizadas en el entrenamiento y en la prueba de las redes. 
Los modelos consisten en un navío de tipo Balizador, una Corveta y una Fragata. Es importante resaltar que, vistas superiores o con ángulos arriba del plano horizontal (Fig. 5-h), no retratan la realidad de un sistema de captura de imágenes de un navío. A través de un análisis previo, fue constatada una baja correlación entre las variables de entrada y salida. De este modo, se optó por la utilización de tres ángulos que caracterizasen situaciones bien distintas para mejor analizar la dependencia de los resultados en relación a la posición angular y verificar que la función de salida es no lineal o de difícil mapeo. Por tanto, para la metodología presentada, los resultados obtenidos fueron basados en el procesamiento de veinticinco diferentes imágenes (diferentes ampliaciones) de los tres tipos de embarcaciones, para tres posiciones angulares distintas $0^{\circ}, 90^{\circ}$ y $150^{\circ}$, generando un total de doscientas y veinticinco imágenes.

Para cada embarcación, fueron obtenidas imágenes de diferentes vistas angulares en perspectivas en torno a $360^{\circ}$. Las diferencias de simetría de las siluetas entre los ángulos dispuestos simétricamente en relación al plano frontal no son diferenciadas por las características extraídas, con excepción del ángulo de dirección del alargamiento del objeto identificado, lo que permite el análisis de reconocimiento apenas de imágenes cuyos ángulos están entre $0^{\circ}$ y $180^{\circ}$ (Fig. $5(\mathrm{a}-\mathrm{g})$ ).

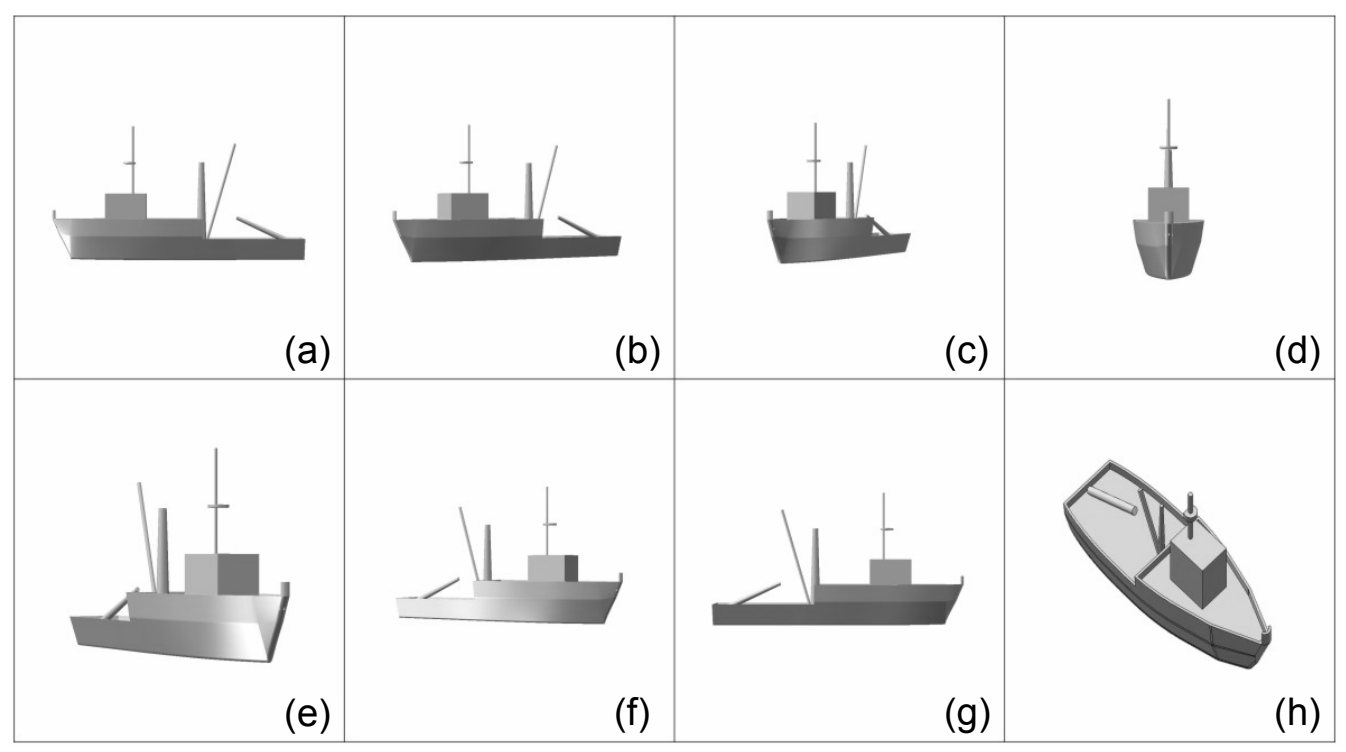

Fig. 5: Vistas angulares del modelo de un navío Balizador en perspectivas (a-g: plano horizontal; h: plano con inclinación).

Por tanto, para cada embarcación fueron escogidas imágenes de apenas tres diferentes vistas angulares en perspectiva. Cada una de las imágenes generadas, referentes a una determinada posición angular, fue convertida para tonos de gris y posteriormente transformada en una imagen con color negro (embarcación) y blanco (fondo). En la Fig. 6, están presentadas las imágenes después del pre-procesamiento de las vistas generadas a partir de los modelos sólidos. Para cada posición angular fueron generadas veinticinco imágenes referentes a diferentes ampliaciones y posteriormente fue hecho el pre-procesamiento resultando un total de setenta y cinco imágenes segmentadas.

La Fig. 7 presenta el proceso de extracción de características a partir del modelo sólido. O sea, para cada embarcación fueron capturadas veinticinco ampliaciones de cada posición angular $\left(0^{\circ}, 90^{\circ} \mathrm{y}\right.$ $\left.150^{\circ}\right)$, y para cada imagen fueron extraídas ocho características geométricas.

Para extracción de las características, fueron desarrollados algoritmos ejecutados en MATLABß que almacena las imágenes de las embarcaciones en estructuras matriciales bidimensionales para cada posición angular considerando las veinticinco diferentes ampliaciones. A partir de estas estructuras, fueron montadas tablas con veintidós conjuntos de características por embarcación para entrenamiento de las topologías de redes y nueve conjuntos para test. 


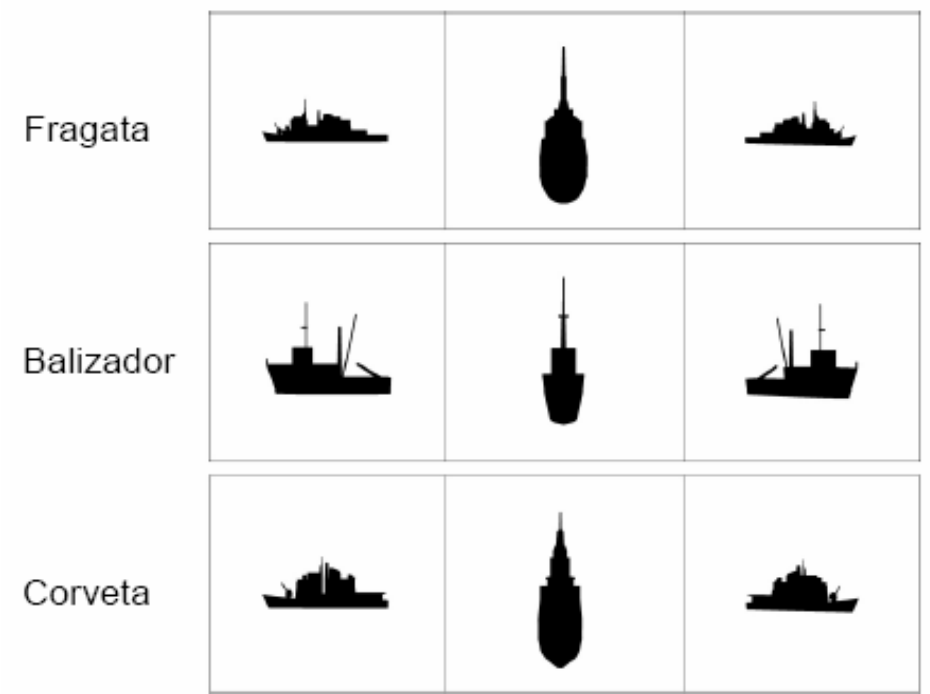

Fig. 6: Imágenes de las vistas angulares después del pre-procesamiento.

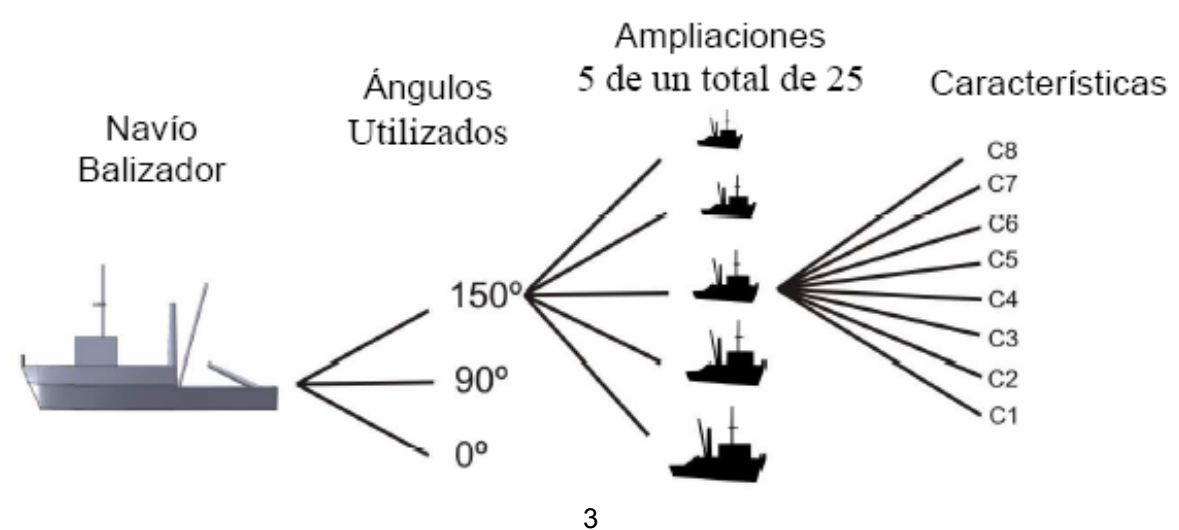

Fig. 7: Esquema de reproducción de imágenes a partir del modelo sólido.

Las características geométricas utilizadas son:

\section{C1 - Área \\ $\mathrm{C} 2-\mathrm{L}_{\max }$ (largo máximo) \\ $\mathrm{C} 3-\mathrm{H}_{\max }$ (altura máxima) \\ C4 - Perímetro}

De pose de las tablas con las características extraídas, los valores son escalonados para la siguiente etapa, que constituye en el entrenamiento y test de diferentes arquitecturas de redes neurales conforme presenta el ejemplo en la Fig. 8.

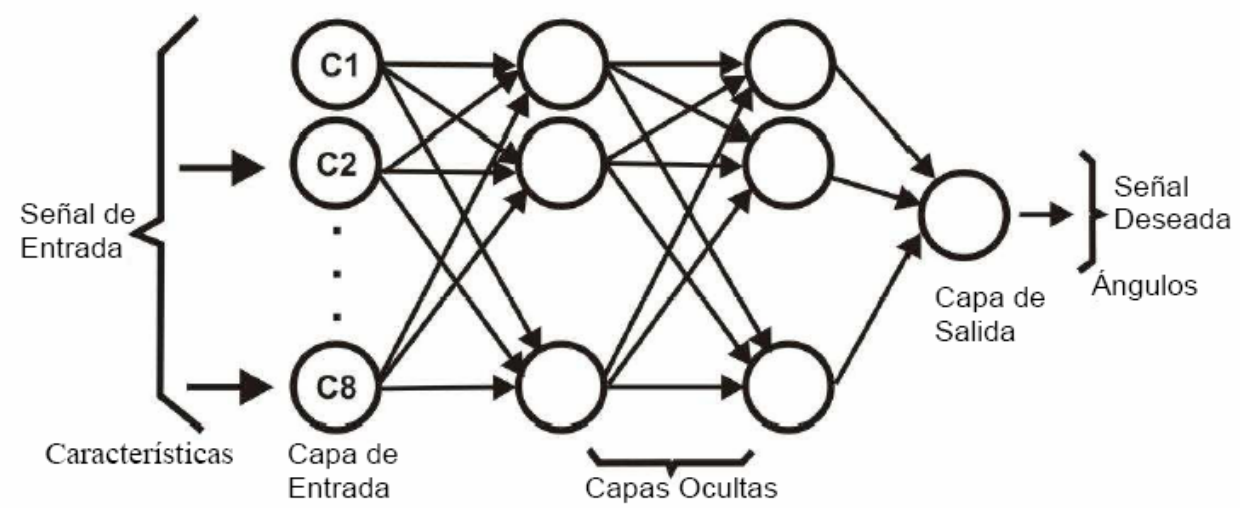

Fig. 8: Ejemplo de Estructura de la Red Neuronal utilizada. 
El modelo de red neuronal artificial será del tipo perceptron (con una o con dos capas ocultas) y función de activación del tipo sigmoidal (Haykin, 1999). Para cada modelo de red (1 y 2 capas) fueron testadas diferentes cantidades de neuronas conforme presentado en la Tabla 1.

Tabla 1: Topologías de redes utilizadas.

\begin{tabular}{|c|c|c|c|c|c|c|c|c|c|c|c|c|}
\hline \multicolumn{13}{|c|}{ Topología de Redes } \\
\hline Identificación & 1 & 2 & 3 & 4 & 5 & 6 & 7 & 8 & 9 & 10 & 11 & 12 \\
\hline $\mathrm{N}^{\circ}$ de Capas Ocultas & 1 & 1 & 1 & 1 & 2 & 2 & 2 & 2 & 2 & 2 & 2 & 2 \\
\hline $\mathrm{N}^{\circ}$ de Nudos de la $1^{\mathrm{a}}$ Capa Oculta & 2 & 5 & 10 & 20 & 2 & 5 & 2 & 5 & 10 & 10 & 20 & 20 \\
\hline $\mathrm{N}^{\circ}$ de Nudos de la $2^{\mathrm{a}}$ Capa Oculta & - & - & - & - & 2 & 2 & 5 & 5 & 5 & 10 & 10 & 20 \\
\hline
\end{tabular}

Para probar las redes fue utilizado el algoritmo backpropagation (Riedmiller, 1993). Los modelos de las redes fueron entrenados para el conjunto de características Geométricas presentadas y considerando un único modelo de embarcación, o sea, después del proceso de su identificación. De un total de setenta y cinco conjuntos de características, esto es, veinticinco conjuntos para cada uno de los tres ángulos escogidos y referentes a las veinticinco posiciones de ampliaciones, fueron estipulados aleatoriamente sesenta y seis conjuntos de características para entrenar cada red y nueve conjuntos para probarlas.

Por tanto, fue hecho un total de treinta y seis entrenamientos para las tres embarcaciones. El resultado de cada test fue analizado y posteriormente computado para cada modelo de embarcación y utilizado en la evaluación de la propuesta del trabajo, esto es, verificación de la posibilidad de reconocimiento de la posición angular del sentido de desplazamiento de la embarcación.

\section{RESULTADOS}

Conforme fue mencionado, las características extraídas fueron montadas en tablas para las diferentes posiciones de ampliación, con cada posición angular codificada como 001 para posición $0^{\circ}, 010$ para $90^{\circ}$ e 100 para $150^{\circ}$, componiendo tres conjuntos de entrenamiento para las topologías de redes presentadas, una para cada embarcación, y más tres conjuntos de pruebas.

Después del entrenamiento de las topologías de redes a partir de las características geométricas, el resultado del test y presentado en la Tabla 2, considerando una tolerancia de 10\%. La Tabla 2 presenta todos los resultados referentes a todas las pruebas realizadas con todos los modelos de rede, todas las tablas de características para cada una de las embarcaciones, esto es, para el Navío Balizador, para la Corveta y para la Fragata.

Tabla 2: Resultados de los modelos de redes para el Navío Balizador, Corveta y Fragata

\begin{tabular}{|c|c|c|c|c|c|c|}
\hline \multicolumn{9}{|c|}{ Resultado general de Redes } \\
\hline \multirow{2}{*}{ Redes } & \multicolumn{2}{|c|}{ Balizador } & \multicolumn{2}{c|}{ Fragata } & \multicolumn{2}{c|}{ Corveta } \\
\cline { 2 - 7 } & Aciertos & Errores & Aciertos & Errores & Aciertos & Errores \\
\hline 1 & 6 & 3 & 4 & 5 & 2 & 7 \\
\hline 2 & 6 & 3 & 8 & 1 & 2 & 7 \\
\hline 3 & 5 & 4 & 8 & 1 & 3 & 6 \\
\hline 4 & 5 & 4 & 8 & 1 & 3 & 6 \\
\hline 5 & 8 & 1 & 9 & 0 & 2 & 7 \\
\hline 6 & 8 & 1 & 9 & 0 & 4 & 5 \\
\hline 7 & 7 & 2 & 8 & 1 & 3 & 6 \\
\hline 8 & 8 & 1 & 8 & 1 & 4 & 5 \\
\hline 9 & 8 & 1 & 8 & 1 & 3 & 6 \\
\hline 10 & 8 & 1 & 8 & 1 & 3 & 6 \\
\hline 11 & 8 & 1 & 8 & 1 & 4 & 5 \\
\hline 12 & 8 & 1 & 8 & 1 & 4 & 5 \\
\hline
\end{tabular}


Observando los resultados presentados, se puede verificar que la topología de red neural 6 para obtención de la posición angular del sentido de desplazamiento, obtuvo un comportamiento mejor que los otros modelos para las tres embarcaciones. Se nota también que las topologías 1 y 2 , con menor número de neuronas, obtuvieron el peor resultado para las tres embarcaciones en media.

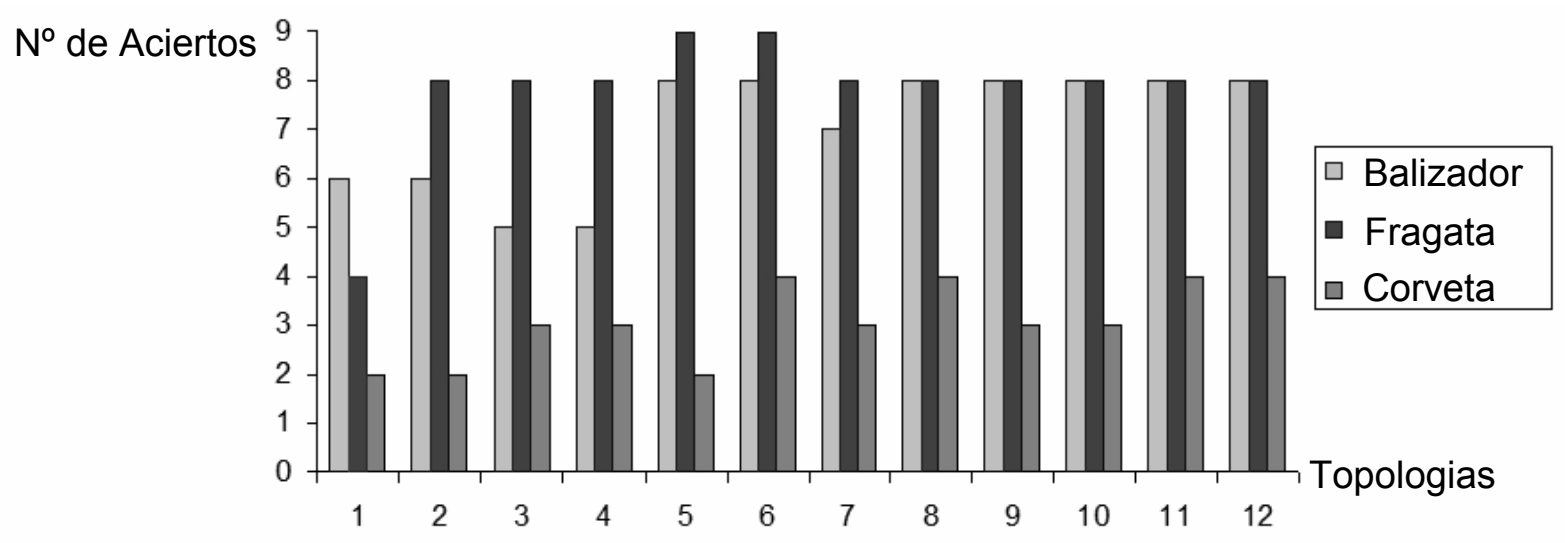

Fig. 9: Gráfico de aciertos para cada navío.

Considerando los datos presentados, se puede afirmar que las topologías de redes neurales que utilizan función de transferencia sigmoidea en las capas escondidas no garantizan mejores resultados para todas las embarcaciones, ya que algunas de las topologías presentaron mejor estabilidad apenas para el navío Balizador y para la Fragata. También se observa que en la mayoría de los casos, las redes con dos capas obtuvieron resultados mejores que con una capa. En la Fig. 9, se puede observar que tanto el navío Balizador como la Fragata, presentaron resultados mejores que los de la Corveta.

\section{CONCLUSIONES}

Para el sistema de detección angular de embarcaciones vía redes neuronales, tanto el número de capas ocultas como el número de neuronas de las capas presentan influencia en la tasa de acierto para la arquitectura utilizada en esta aplicación. Entonces, dependiendo de la topología que será utilizada, se puede obtener una mejor o peor tasa de acierto para detección del ángulo. También fue observado que la metodología no presenta respuestas iguales para todas las embarcaciones, lo que significa que otras técnicas deben ser utilizadas como complemento de los resultados. En el estudio fueron utilizados tres ángulos, porque el objetivo del trabajo es verificar si la función que caracteriza las salidas sería posible de ser mapeada. La dificultad de este mapeo fue constatada a través de los resultados obtenidos, el que apunta para la necesidad de ampliarse el banco de datos en futuros estudios.

La metodología propuesta analizó apenas los resultados de la fase de reconocimiento y determinación del ángulo, a través de redes neurales y verificó una nueva posibilidad en la mejoría de la eficiencia de una de las etapas involucradas en el proyecto desarrollado por el Instituto de Pesquisas de la Marina, de un clasificador basado en imágenes infrarrojas de navíos. A cada instante, nuevos desafíos surgen como fomento de estudios, como es el caso específico de la utilización de imágenes infrarrojas, donde las dificultades están desde el costo de la captura como a la interpretación de las imágenes en función de la calidad, en relación al nivel y al tipo de ruido. En función de estos factores, los desafíos, aún en niveles experimentales, prometen muchos trabajos futuros de importante interés para la sociedad.

Teniendo en cuenta que, para el reconocimiento en aplicaciones semejantes, muchos autores utilizan los momentos de $\mathrm{Hu}$ en la identificación de diversos tipos de objetos, se sugiere, como futuros trabajos, un análisis comparativo de los resultados obtenidos a través del conjunto de características presentado con referencia a otros grupos de características. Para las topologías de redes, también se sugiere que la sustitución de la función de activación sigmoidal por la función tangente hiperbólica, deben ser llevadas en consideración en futuros trabajos, así como la utilización 
de otros sistemas de reconocimiento como, por ejemplo, un sistema clasificador o máquinas de vector suporte (SVM) para el reconocimiento.

\section{AGRADECIMIENTOS}

Los autores agradecen a FAPERJ por el apoyo financiero (No. E-26/171.362/2001), CNPq y CAPES / Brasil (Pro Programa de Ingeniería PE021-2008).

\section{REFERENCIAS}

Alves, J. A.; "Recognition of Ship Types from a Infrared Image using Moment Invariants and Neural Networks", Dissertação de Mestrado, Naval Postgraduate School, Monterey, Califórnia, EUA (2001).

Behrooz Kamgar-Parse, y Behzad Kamgar-Parsi; "Improved Image Thresholding for Object Extraction in IR Images", Proceedings of International Conference on Image Processing 2001, 758761, Outubro (2001).

Carvalho, S. R. G.; "Classificação de Técnicas Segmentação de Alvos em Imagens Infravermelhas", Dissertação de Mestrado - UFF/RJ (2008).

Chang K. y J. Ghosh; "Principal curve classifier - a nonlinear approach to pattern classification," IEEE International Joint Conference on Neural Networks, 695-700, May (1998).

Conci, A.,E. Azevedo y F. R. Leta; "Computação Gráfica - Teoria e Prática", [v.2]. Rio de Janeiro. Elsevier Editora Ltda (2008).

Deer, S. Z. y R. Chellapa; "Probe-based Automatic Target Recognition in Infrared Imagery", IEEE Transactions on Image Processing, Vol. 6, 92-102, January (1997).

Feng, Z., L. Shang-qian, W. Da-Bao, G. Wei; "Aircraft recognition in infrared image using wavelet moment invariants". Image and Vision Computing, Elsevier B. V (2008).

Fernandez, H. L.; "Classificação de Navios Baseada em Curvas Principais", Dissertação D.Sc, COPPE/UFRJ, Rio de Janeiro (2005).

Freeman, J. A., y D. M. Skapura; Neural Networks: Algorithms, Applications and Programing Techiniques, Addison-Wesley, Reading, MA: (1992).

Gonçalves Filho, A. do E. L.; Sistema de Reconhecimento de Objetos para Automação Industrial, Dissertação de M.Sc., Programa de Pós-Graduação em Engenharia Mecânica, PUC-Rio, (1996).

Haykin, S.; “Neural Networks: A Comprehensive Foundation”. Prentice Hall, (1999).

John Russ C.; "The Image Processing Handbook”, CRC Press - IEEE Press, 3 edition (1998).

Neves, S. R.; “Algoritmos para Segmentação de Imagens Infravermelhas”, Dissertação de D.Sc., COPPE/UFRJ, Rio de Janeiro, RJ, Brasil (2005).

Neves S. R., E. A. B. Silva y G. V. Mendonça; "Wavelet-watershed automatic infrared image segmentation method", Electronic Letters, vol. 39, no. 12, 903-904, June (2003).

Riedmiller M. y H. Brown; “A Direct adaptive method for faster backpropagation learning: The RPROP algorithm", Proceedings of the IEEE International Conference on Neural Networks, (1993).

Ruiz E. V.; "Aprendizaje y Percepción: Preproceso y Extracción de Características" Facultad de Informática-Universidad Politécnica de Valencia, Febrero (2001).

Valencia, P. E.; "Clasificación de patrones mediante redes neuronales artificiales", Anales del instituto de ingenieros de Chile, 24-31, Abril (1999). 